\title{
A sub 1V CMOS bandgap reference with two diodes
}

\author{
Hyung-Jin Choi ${ }^{1}$, Jeong $\mathrm{Cho}^{2}$ and Soo-Won Kim ${ }^{2}$ \\ ${ }^{1}$ Department of Nano-Semiconductor Engineering, Korea University, Seoul, Republic of Korea \\ ${ }^{2}$ Department of Electrical Engineering, Korea University, Seoul, Republic of Korea
}

\begin{abstract}
A small size, sub 1V CMOS bandgap voltage reference with the temperature coefficient (TC) of $12 \mathrm{ppm} /{ }^{\circ} \mathrm{C}$ from $0^{\circ} \mathrm{C}$ to $100^{\circ} \mathrm{C}$ is presented in this paper. The proposed circuit offers several performance advantages over previous bandgap voltage reference circuits. Compared to a conventional structure, it achieves a smaller area by using only two diodes. Also, it can fulfil sub $1 \mathrm{~V}$ reference voltage by using temperature coefficient of threshold voltage to generate a current proportional to absolute temperature. This is because $\mathrm{TC}$ of this current on proposed circuit is bigger than that of conventional structure. Detailed analysis of the proposed bandgap reference is provided, which is simulated using $0.35 \mu \mathrm{m}$ CMOS process. The proposed bandgap reference occupies an active area of $0.0067 \mathrm{~mm}^{2}$. The simulation results are the voltage reference output voltage of $0.843 \mathrm{~V}$ and an accuracy of $\pm 0.08 \%$.
\end{abstract}

\section{Introduction}

Voltage reference is at most essential part on mixedsignal applications, such as analog-to-digital (AD) and digital-to-analog (D/A) converters, regulators. Generally, precision of voltage reference over supply voltage and operating temperature is important factor, because it limits the resolution of these applications. So bandgap reference which makes voltage reference less sensitive to temperature is important. Recently, the demand for small size and low power is increasing. As a result, the demand for bandgap references which have small size and sub $1 \mathrm{~V}$ voltage reference is also increasing. For size of bandgap reference, the number of diodes is the critical factor, because diodes are implemented by bipolar transistor transistors whose size is very huge. Thus, the number of diodes must be decreased to reduce active area. The conventional bandgap references generally use more than 9 diodes, leading to large die area. And they have voltage reference range from $1.1 \mathrm{~V}$ to $1.3 \mathrm{~V}[1,2]$. This is because of low positive temperature coefficient of current. Sub 1$\mathrm{V}$ bandgap voltage references are described in [3-8]. However, since these bandgap references have complex structure or have high current, they occupy large area or consume high power.

A sub-1V bandgap reference (BGR) with a small area is presented in this paper. The following chapters show conventional BGR structure and proposed BGR structure including mathematical analysis and the simulation results.

\section{Conventional bandgap reference}

Generally, BGR uses negative temperature coefficient (TC) and positive TC. These two TCs counterbalance each other. So, it can make voltage reference independent to temperature. Figure 1 shows the schematic of conventional BGR. It uses base-emitter voltage of bipolar transistors or, forward bias voltage of a diode to make a negative (TC). That negative TC value is approximatively $1.5 \mathrm{mV} /{ }^{\circ} \mathrm{C}$, is explained in $[9,10]$. Conventional BGR also uses difference between forward voltages of diodes to make positive TC, when current flowing diodes is same. Because their difference is proportional to the absolute temperature (PTAT). Figure 2 shows concept of difference between forward voltages of diodes, and equation of PTAT voltage is given by (1).

$$
\begin{aligned}
\Delta V_{D} & =V_{D 1}-V_{D 2} . \\
& =V_{T} \ln \frac{I}{I_{O}}-V_{T} \ln \frac{I}{n I_{O}} . \\
& =V_{T} \ln n . \\
& \left(I_{O} \text { is saturation current of diode }\right) \\
& (n \text { is number of diode D2 in Fig. } 2)
\end{aligned}
$$

$V_{T}$ is proportional to temperature, and is presented in (2).

$$
\begin{aligned}
V_{T} & =k T / q . \\
& (k \text { is Boltzmann constant }) \\
& (T \text { is absolute temperature }) \\
& (q \text { is quantity of electric charge) }
\end{aligned}
$$

So, difference between forward voltages of diodes has positive TC. Conventional BGR uses both negative TC and positive TC to make voltage reference independent to temperature. Amplifier in Fig. 1 makes node voltage of A 
equal to $V_{D 2}$. Also, amplifier makes current flowing D2 equal to current flowing D1. This is because output of amplifier is input of $p$ type mosfets, which have same width and length. As a result, voltage drop across R1 is difference between forward voltages of diodes and has positive TC. Current flowing R1 is given by

$$
\begin{aligned}
I & =\frac{V_{D 1}-V_{D 2}}{R 1} . \\
& =\frac{1}{R 1}\left(V_{T} \ln \frac{n I}{I_{O}}-V_{T} \ln \frac{I}{I_{O}}\right) . \\
& =\frac{V_{T}}{R 1} \ln n .
\end{aligned}
$$

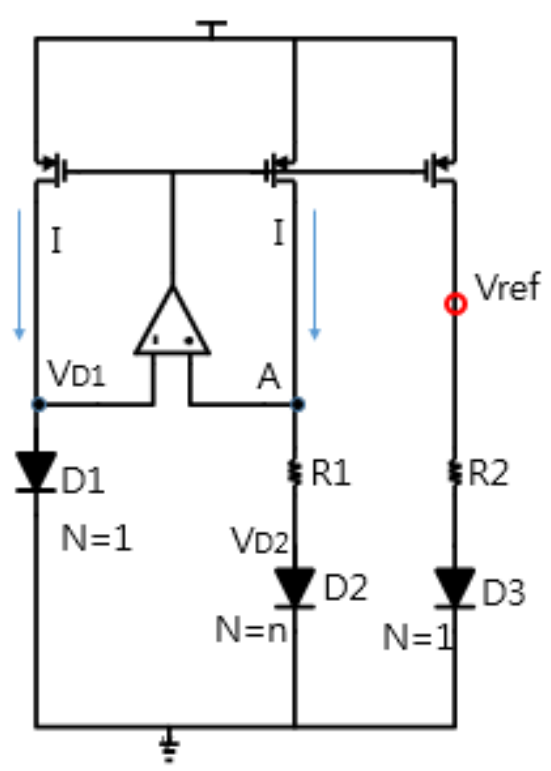

Figure 1. Schematic of the conventional bandgap reference.

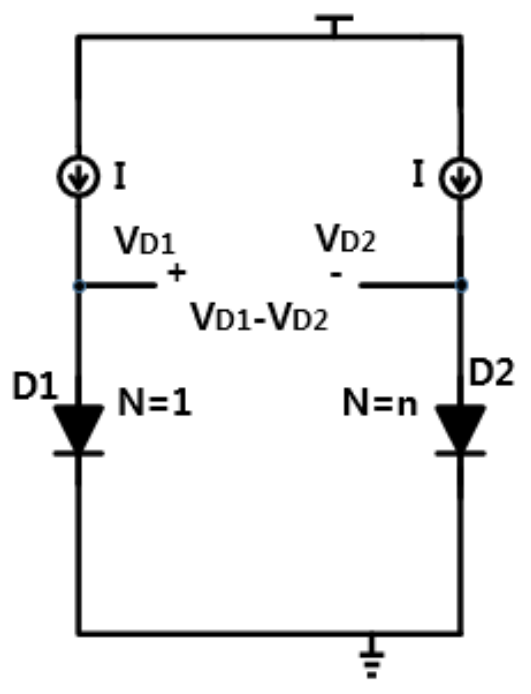

Figure 2. Conceptual schematic of PTAT voltage.

Eq. (3) shows that the current flowing R1 $(I)$ is growing up by increasing temperature, because difference between forward voltages of diodes is PTAT. P type mosfets copy the current flowing R2 from that of R1. So voltage drop across R2 is also PTAT. As a result, $V_{r e f}$ is given by,

$$
\begin{aligned}
V_{r e f} & =V_{D 3}+R_{2} I . \\
& =V_{D 3}+\frac{R_{2}}{R_{1}} V_{T} \ln n .
\end{aligned}
$$

$V_{D 3}$ is turn on voltage of $\mathrm{D} 3$, which has negative TC, while voltage drop across R2 is PTAT. So both negative TC and positive TC counterbalance each other. TC of $V_{\text {ref }}$ is represent by

$$
\begin{aligned}
\frac{\partial V_{r e f}}{\partial T} & =\frac{\partial V_{D 3}}{\partial T}+R_{2} \frac{\partial V_{T}}{\partial T} \ln n . \\
& =\frac{\partial V_{D 3}}{\partial T}+\frac{R_{2}}{R_{1}} \frac{k}{q} \ln n=0 .
\end{aligned}
$$

( $n$ is number of D2/number of D1)

Therefore, $V_{\text {ref }}$ can be independent on temperature.

\section{Proposed bandgap reference}

Figure 3 shows the schematic of proposed BGR. It only uses two diode (D1, D2). Proposed circuit uses forward bias diode, which has negative TC when the current flowing diode D1 is fixed. And, it also uses current flowing $\mathrm{R} 1$, which has positive TC. It is possible to analyse positive TC of current flowing R1 by using property that currents flowing $\mathrm{M} 1$ and $\mathrm{R} 1$ in Fig. 3 are same, Since R1 and M1 are connected in series. The amount of current flowing them is same as

$$
\frac{1}{2} u_{p} C_{o x} \frac{W}{L}\left(V_{D D}-A-V_{t h}\right)^{2}=\frac{V_{A}-V_{B}}{R_{1}} .
$$

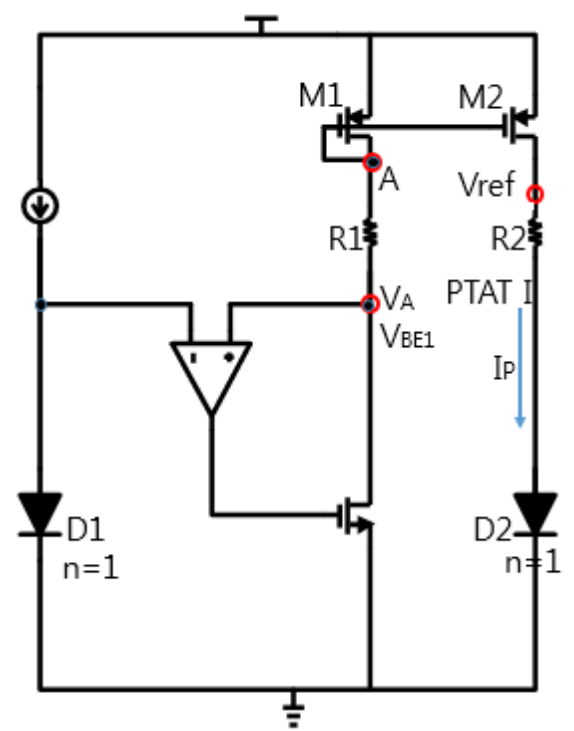

Figure 3. Schematic of the proposed bandgap reference.

If the current flowing R1 in Fig. 3 is analysed about temperature, temperature variation of current on $\mathrm{R} 1$ is given by (7). 


$$
\begin{gathered}
u_{p} C_{o x} \frac{W}{L}\left(V_{D D}-A-V_{t h}\right)\left(-\frac{\partial A}{\partial T}-\frac{\partial V_{t h}}{\partial T}\right)=\frac{1}{R_{1}}\left(\frac{\partial A}{\partial T}-\frac{\partial V_{A}}{\partial T}\right) \\
\frac{\partial A}{\partial T}=\frac{\frac{1}{R_{1}}}{\frac{1}{R_{1}}+g_{m p}} \frac{\partial V_{A}}{\partial T}-\frac{g_{m p}}{\frac{1}{R_{1}}+g_{m p}} \frac{\partial V_{t h}}{\partial T} . \\
\frac{\partial I_{P}}{\partial T}=\frac{1}{R_{1}}\left(\frac{\partial A}{\partial T}-\frac{\partial V_{A}}{\partial T}\right) \\
=\frac{1}{R_{1}}\left(\frac{\frac{W}{R}}{\frac{1}{R}+V_{m}} \frac{\partial V_{A}}{\partial T}-\frac{g_{m}}{\frac{1}{R}+g_{m}} \frac{\partial V_{t h}}{\partial T}-\frac{\partial V_{A}}{\partial T}\right) . \\
=-\frac{1}{R_{1}}\left(\frac{g_{m}}{\frac{1}{R_{1}}+g_{m}}\right)\left(\frac{\partial V_{A}}{\partial T}+\frac{\partial V_{t h}}{\partial T}\right) .
\end{gathered}
$$

Eq. (7) shows that the current flowing $\mathrm{R} 1\left(I_{p}\right)$ is growing up by increasing temperature, because $V_{A}$ and $V_{t h}$ have negative TC. As a result, $I_{P}$ is PTAT. P type mosfets copy the current flowing $\mathrm{R} 2$ from that of R1. Reference voltage $V_{\text {ref }}$ is represented by

$$
V_{\text {ref }}=V_{D 2}+R_{2} I_{P}
$$

which $V_{D 2}$ is turn-on voltage of D2. When the temperature increases, $V_{D 2}$ decreases due to the negative $\mathrm{TC}$ of $V_{D 2}$, while the voltage drop across $\mathrm{R} 2$ increases due to the increase of $I_{P}$. TC of $V_{r e f}$ is given by

$$
\begin{aligned}
\frac{\partial V_{r e f}}{\partial T} & =\frac{\partial V_{D 2}}{\partial T}+R_{2} \frac{\partial I_{P}}{\partial T} . \\
& =\frac{\partial V_{D 2}}{\partial T}-\frac{R_{2}}{R_{1}}\left(\frac{g_{m}}{\frac{1}{R_{1}}+g_{m}}\right)\left(\frac{\partial V_{A}}{\partial T}+\frac{\partial V_{t h}}{\partial T}\right) . \\
& =\frac{\partial V_{D 2}}{\partial T}-\frac{R_{2}}{R_{1}}\left(\frac{g_{m}}{\frac{1}{R_{1}}+g_{m}}\right)\left(\frac{\partial V_{D 1}}{\partial T}+\frac{\partial V_{t h}}{\partial T}\right)=0 .
\end{aligned}
$$

As the temperature goes up, increasing of voltage drop across R2 counterbalances decreasing at $V_{D 2}$. Therefore, $V_{\text {ref }}$ can be independent on temperature.

The PTAT current TC of proposed BGR is bigger than that of conventional structure [1], when amount of PTAT currents is same. Thus, when PTAT current counterbalances negative TC of $V_{D 2}$, amount of PTAT current in proposed BGR is smaller than that of conventional structure. As a result, voltage drop across $\mathrm{R} 2$ is also smaller than that of conventional art, and $V_{\text {ref }}$ is low voltage, which is under the $1 \mathrm{~V}$.

\section{Use BMR for current source}

A beta-multiplier reference (BMR) insensitive to supply voltage variation, is used as the current source in bandgap reference as shown in Figure 4. The BMR current is given by (10).

$$
\begin{aligned}
& I_{C}=\frac{2}{u_{n} C_{o x}(W / L)_{N}} \cdot \frac{1}{R_{S}{ }^{2}}\left(1-\frac{1}{\sqrt{K}}\right)^{2} . \\
& =\frac{2}{u_{n} C_{o x}(W / L)_{N}} \cdot \frac{1}{R_{S}{ }^{2}}\left(1-\frac{1}{\sqrt{K}}\right)^{2}=\frac{A}{u_{n}} . \\
& \left(A=\frac{2}{C_{o x}(W / L)_{N}} \cdot \frac{1}{R_{S}{ }^{2}}\left(1-\frac{1}{\sqrt{K}}\right)^{2}\right)
\end{aligned}
$$

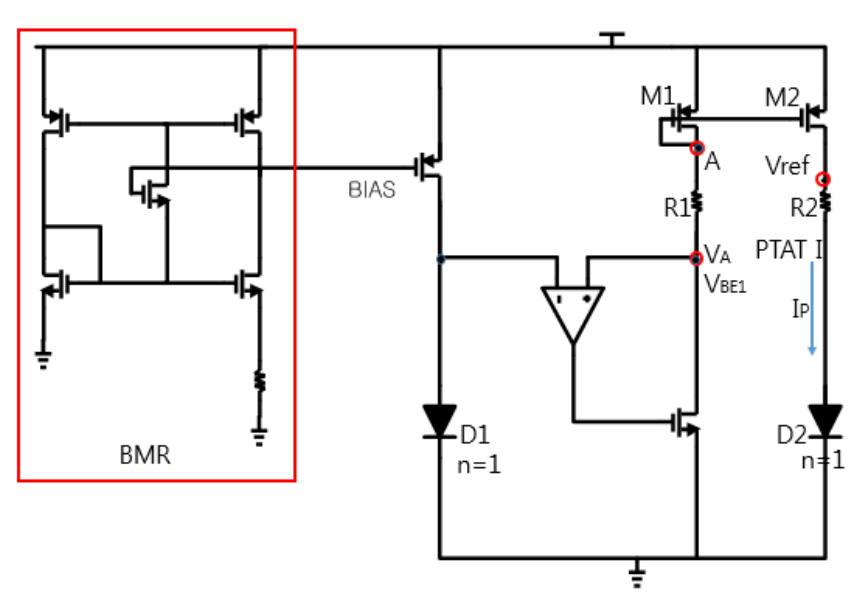

Figure 4. Proposed BGR with BMR.

However The BMR current is affected according to temperature variation. Since this effect can vary TC of turn-on voltage of diode $\left(V_{D}\right)$, TC of BMR current must be considered when TC of $V_{D}$ is analysed. TC of BMR current is given by

$$
\frac{\partial I}{\partial T}=-A \frac{1}{u_{n}^{2}} \frac{\partial u_{n}}{\partial T} .
$$

TC of $V_{D}$ considering TC of BMR current is given by

$$
\begin{aligned}
\frac{\partial V_{B E}}{\partial T} & =\frac{\partial V_{T}}{\partial T} \ln \frac{I_{C}}{I_{O}}+V_{T}\left(\frac{1}{I_{C}} \frac{\partial I_{C}}{\partial T}-\frac{1}{I_{O}} \frac{\partial I_{O}}{\partial T}\right) . \\
& =\frac{\partial V_{T}}{\partial T} \ln \frac{I_{C}}{I_{O}}-\frac{V_{T}}{u_{n}} \frac{\partial u_{n}}{\partial T}-\frac{V_{T}}{I_{O}} \frac{\partial I_{O}}{\partial T} . \\
& =\left(\frac{\partial V_{T}}{\partial T} \ln \frac{I_{C}}{I_{O}}-\frac{V_{T}}{I_{O}} \frac{\partial I_{O}}{\partial T}\right)-\frac{V_{T}}{u_{n}} \frac{\partial u_{n}}{\partial T} . \\
& =\frac{V_{B E}-(4+m) V_{T}-E_{g} / q}{T}-\frac{V_{T}}{u_{n}} \frac{\partial u_{n}}{\partial T} . \\
& \approx \frac{V_{B E}-(4+m) V_{T}-E_{g} / q}{T} .
\end{aligned}
$$

( $I_{O}$ is saturation current of diode)

Eq. (12) shows that temperature variation of BMR current has no impact on temperature coefficient of turn on voltage of diode at all.

\section{Simulation Results}


The proposed BGR and conventional BGR are designed and simulated with $0.35 \mu \mathrm{m}$ CMOS process. Layout view of Conventional BGR is presented in Figure 5. It has large active area, which is $0.0132 \mathrm{~mm}^{2}$. Figure 6 shows a layout view of the proposed BGR. It occupies an active area of $0.0067 \mathrm{~mm}^{2}$, which is smaller than of [1], [2] and [3]. Conventional BGR approximatively has twice area compared to area of proposed BGR.

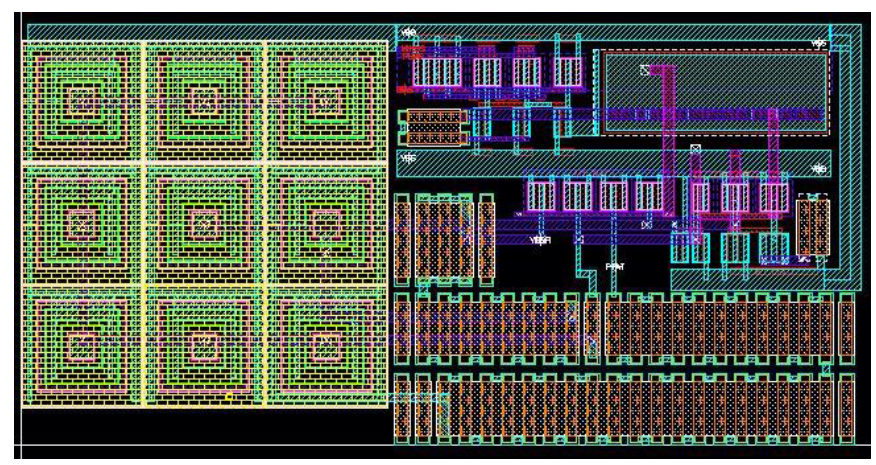

Figure 5. Layout view of the conventional BGR..

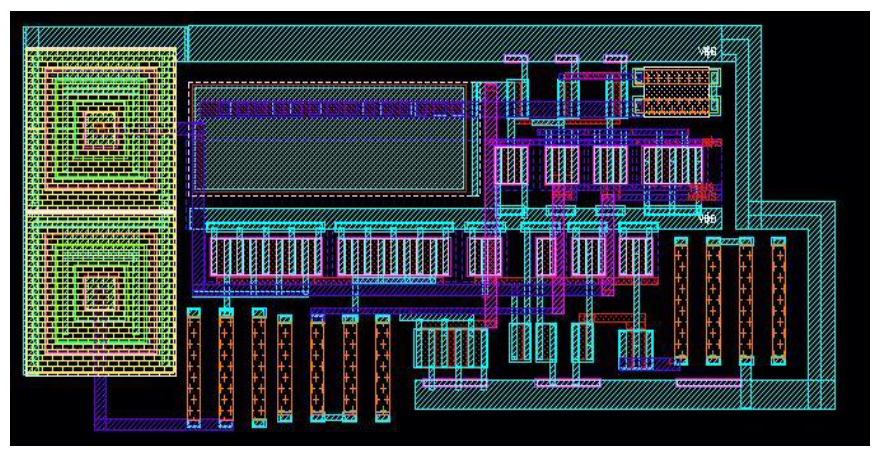

Figure 6. Layout view of the proposed BGR.

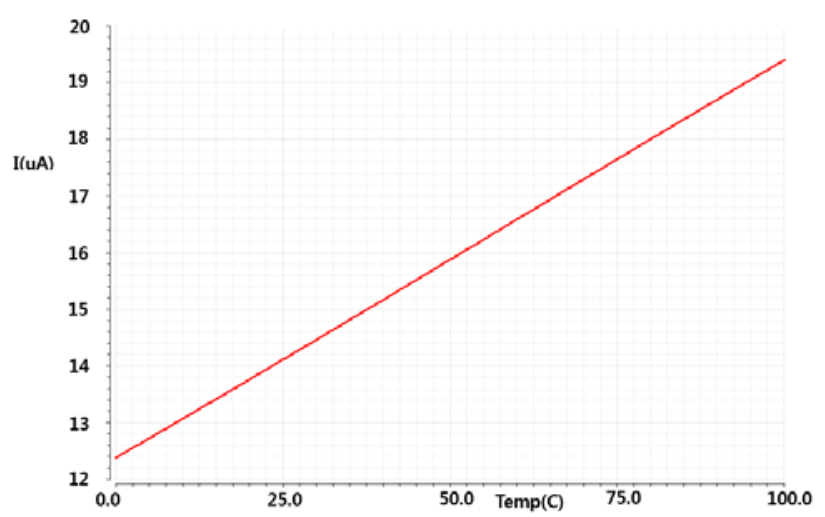

Figure 7. Simulation result of $I$

TC of PTAT current $I$ in conventional bandgap at Fig 1 is $0.7 \mathrm{nA} /{ }^{\circ} \mathrm{C}$, which is described in Figure 7. It is measured when $I$ at $0^{\circ} \mathrm{C}$ is overall $12.4 \mathrm{uA}$. Whereas, TC of PTAT current $I_{P T A T}$ in proposed BGR is $1.65 \mathrm{nA} /{ }^{\circ} \mathrm{C}$, as shown in Figure 8 . This TC value is also measured when $I_{P T A T}$ at $0^{\circ} \mathrm{C}$ is overall $12.4 \mathrm{uA}$. Proposed BGR has bigger TC value of PTAT current than that of conventional BGR. TC of PTAP current in proposed BGR is 2.35 times larger than that of conventional BGR, which is the same result expected in the previous chapter. A slope of $I_{P T A T}$ can be changed by adjusting $\mathrm{W} / \mathrm{L}$ ratio of $\mathrm{M} 1$ and $\mathrm{M} 2$. Conventional BGR output voltage of bandgap reference is represented in Figure 9. Output voltage $V_{R E F}$ is $1.168 \mathrm{~V}$, which is larger than $1 \mathrm{~V}$. Peak to peak error for voltage is $1.2 \mathrm{mV}$, and $\mathrm{TC}$ is $12 \mathrm{ppm} /{ }^{\circ} \mathrm{C}$ in the temperature range from $0^{\circ} \mathrm{C}$ to $100^{\circ} \mathrm{C}$. Figure 10 shows output voltage of proposed BGR. Output voltage of proposed BGR $V_{R E F}$ is $0.843 \mathrm{~V}$. The maximum voltage variation and the $\mathrm{TC}$ are $1.2 \mathrm{mV}$ and $12 \mathrm{ppm} /{ }^{\circ} \mathrm{C}$ in the temperature range from $0{ }^{\circ} \mathrm{C}$ to $100^{\circ} \mathrm{C}$, respectively. Proposed BGR has almost same precision for temperature variation compared to conventional BGR, but it has small reference voltage that is sub $1 \mathrm{~V}$

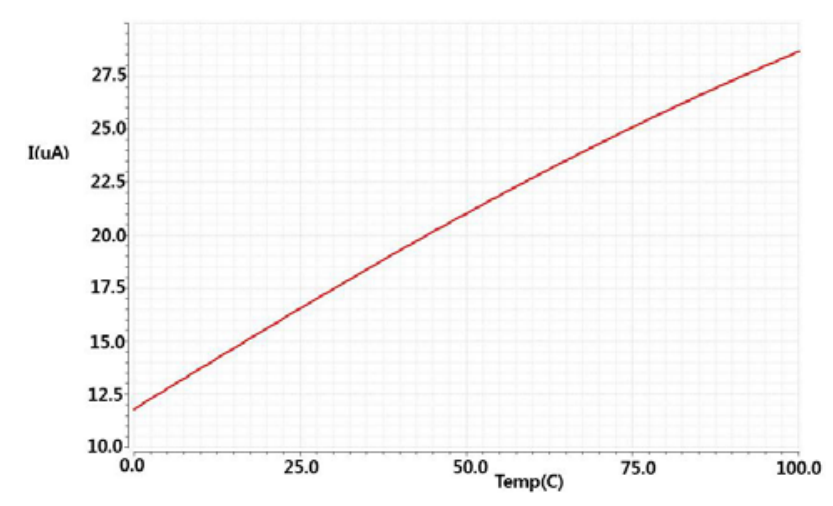

Figure 8. Simulation result of $I_{P T A T}$.

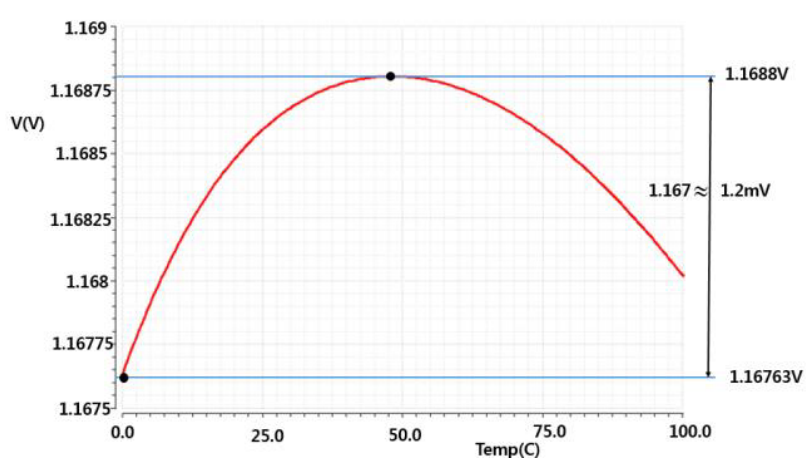

Figure 9. Simulation result of conventional BGR $V_{R E F}$.

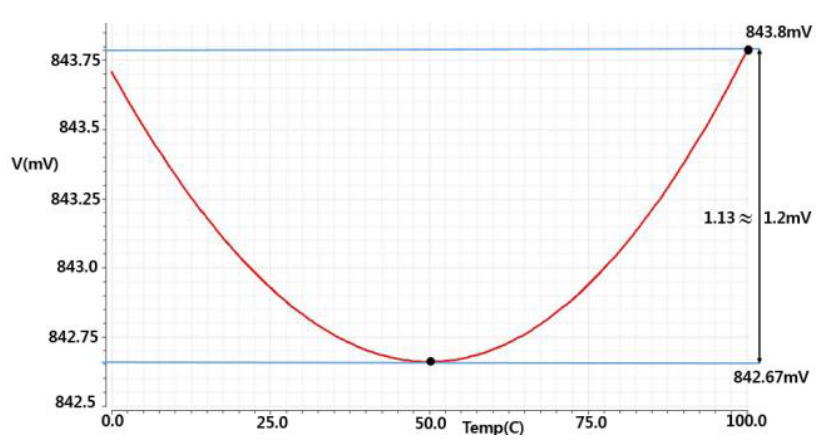

Figure 10. Simulation result of proposed BGR $V_{R E F}$.

\section{Conclusion}

This paper presents the CMOS bandgap reference with small size and sub $1-\mathrm{V}$ operation by using only two diode and TC of Vth to make PTAT current, respectively. It occupies an active area of $0.0067 \mathrm{~mm}^{2}$. The simulation 
results are the voltage reference output of $0.843 \mathrm{~V}$, the accuracy of $\pm 0.08 \%$, and $\mathrm{TC}$ of $12 \mathrm{ppm} /{ }^{\circ} \mathrm{C}$ from $0{ }^{\circ} \mathrm{C}$ to $100^{\circ} \mathrm{C}$.

\section{Acknowledgement}

This work was partly supported by the IT R\&D program of MSIP/IITP [2014-050-005-038, Development of Fast Wireless Charge Technology for 2A Mobile Device Supporting Dual Standard].

\section{References}

1. P. R. Gray and R. G. Meyer, Analysis and Design of Analog Integrated Circuits. New York :Wiley pp. 289-296 (1977),

2. R. J. Widlar, "New developments in IC voltage regulators," IEEE J.Solid-state Circuits, vol. 6, pp. 27, Feb. (1971)

3. Banba, Hironori, et al. "A CMOS bandgap reference circuit with sub-1-V operation." Solid-State Circuits, IEEE Journal of 34.5: 670-674 (1999)

4. Sanborn, Keith, Dongsheng Ma, and Vadim Ivanov. "A sub-1-V low-noise bandgap voltage reference." Solid-State Circuits, IEEE Journal of 42.11: 24662481 (2007):

5. Jiang, Yueming, and Edward KF Lee. "Design of low-voltage bandgap reference using transimpedance amplifier." Circuits and Systems II: Analog and Digital Signal Processing, IEEE Transactions on 47.6: 552-555 (2000).

6. Chen, Suheng, and Benjamin J. Blalock. "Switched capacitor bandgap voltage reference for sub-1-V operation." IEICE Electronics Express 3.24: 529-533 (2006).

7. Wiessflecker, Martin, et al. "A sub 1V self clocked switched capacitor bandgap reference with a current consumption of 180nA." Circuits and Systems (ISCAS), 2012 IEEE International Symposium on. IEEE, 2012 : pp 2841-2844(2012).

8. Shrivastava, Aatmesh, et al. "5.4 A 32nW bandgap reference voltage operational from $0.5 \mathrm{~V}$ supply for ultra-low power systems." Solid-State Circuits Conference-(ISSCC), 2015 IEEE International. IEEE, 2015.

9. Ohte, Akira, and Michiaki Yamagata. "A precision silicon transistor thermometer." Instrumentation and Measurement, IEEE Transactions on 26.4: 335-341 (1977).

10. Freire, Raimundo Carlos Silverio, Sergio Daher, and Gurdip Singh Deep. "A highly linear single pn junction temperature sensor." Instrumentation and Measurement, IEEE Transactions on 43.2: 127-132 (1994) 